\title{
Possible spin-orbit driven spin-liquid ground state in the double perovskite phase of $\mathrm{Ba}_{3} \mathrm{YIr}_{2} \mathrm{O}_{9}$
}

\author{
Tusharkanti Dey, ${ }^{1,}{ }^{*}$ A. V. Mahajan, ${ }^{1, \dagger}$ R. Kumar, ${ }^{1}$ B. Koteswararao, ${ }^{2}$ F. C. Chou, ${ }^{2}$ A. A. Omrani, ${ }^{3}$ and H. M. Ronnow ${ }^{3}$ \\ ${ }^{1}$ Department of Physics, Indian Institute of Technology Bombay, Powai, Mumbai 400076, India \\ ${ }^{2}$ Center for Condensed Matter Sciences, National Taiwan University, Taipei 10617, Taiwan \\ ${ }^{3}$ Laboratory for Quantum Magnetism, Ecole Polytechnique Federale de Lausanne (EPFL), CH 1015, Switzerland
} (Received 16 October 2012; revised manuscript received 25 September 2013; published 25 October 2013)

\begin{abstract}
We report the structural transformation of hexagonal $\mathrm{Ba}_{3} \mathrm{YIr}_{2} \mathrm{O}_{9}$ to a cubic double perovskite form (stable in ambient conditions) under an applied pressure of $8 \mathrm{GPa}$ at $1273 \mathrm{~K}$. While the ambient pressure synthesized sample undergoes long-range magnetic ordering at $\sim 4 \mathrm{~K}$, the high-pressure (HP) synthesized sample does not order down to $2 \mathrm{~K}$ as evidenced from our susceptibility, heat capacity, and nuclear magnetic resonance (NMR) measurements. Further, for the HP sample, our heat capacity data have the form $\gamma T+\beta T^{3}$ in the temperature (T) range of $2-10 \mathrm{~K}$ with the Sommerfeld coefficient $\gamma=10 \mathrm{~mJ} / \mathrm{mol}-\mathrm{Ir} \mathrm{K}^{2}$. The ${ }^{89} \mathrm{Y}$ NMR shift has no $T$ dependence in the range of $4-120 \mathrm{~K}$ and its spin-lattice relaxation rate varies linearly with $T$ in the range of 8-45 K (above which it is $T$ independent). Resistance measurements of both the samples confirm that they are semiconducting. Our data provide evidence for the formation of a $5 d$-based, gapless, quantum spin-liquid in the cubic (HP) phase of $\mathrm{Ba}_{3} \mathrm{YIr}_{2} \mathrm{O}_{9}$. In this picture, the $\gamma T$ term in the heat capacity and the linear variation of ${ }^{89} \mathrm{Y} 1 / T_{1}$ arises from excitations out of a spinon Fermi surface. Our findings lend credence to the theoretical suggestion [Chen, Pereira, and Balents, Phys. Rev. B 82, 174440 (2010)] that strong spin-orbit coupling can enhance quantum fluctuations and lead to a QSL state in the double perovskite lattice.
\end{abstract}

DOI: 10.1103/PhysRevB.88.134425

PACS number(s): 75.40.Cx, 71.70.Ej, 76.60.-k

The $3 d$ transition metal oxides have been studied extensively to explore novel properties such as superconductivity, ${ }^{1,2}$ colossal magnetoresistance, ${ }^{3}$ etc. In these materials, the orbital angular momentum of the valence electrons is largely quenched and a large onsite Coulomb energy often drives the materials to a Mott insulating state. In contrast, for $5 d$-group-based materials, this onsite Coulomb energy is smaller by an order of magnitude and one could expect more metallic and less magnetic behavior. However, some $5 d$ Ir-based materials such as $\mathrm{Na}_{4} \mathrm{Ir}_{3} \mathrm{O}_{8},{ }^{4} \mathrm{Sr}_{2} \mathrm{IrO}_{4},{ }^{5,6}$ and $\mathrm{Na}_{2} \mathrm{IrO}_{3}{ }^{7}$ are found to be insulators showing exotic magnetic properties. These materials are insulating due to the presence of strong spin-orbit interactions and are often called spin-orbit driven Mott insulators. Such materials are relatively less explored and expected to offer a promising playground for materials researchers.

Earlier we have investigated hexagonal $\mathrm{Ba}_{3} \operatorname{IrTi}_{2} \mathrm{O}_{9}$ and suggested it to be a spin-orbit driven liquid based on a triangular lattice. ${ }^{8}$ Recently, $\mathrm{Ba}_{3} \mathrm{IrTi}_{2} \mathrm{O}_{9}$ has been proposed as a candidate material to study the Heisenberg-Kitaev model on a triangular lattice. ${ }^{9}$ It will be interesting to explore other iridates having a triangular lattice. $\mathrm{Ba}_{3} \mathrm{YIr}_{2} \mathrm{O}_{9}$ has a similar chemical formula like $\mathrm{Ba}_{3} \operatorname{IrTi}_{2} \mathrm{O}_{9}$ and it crystallizes in the hexagonal structure $\left(\mathrm{P}_{3} / \mathrm{mmc}\right)$ with Ir-Ir structural dimers arranged in an edge-shared triangular fashion..$^{10}$ Since all the Ir are equivalent, they should have a fractional oxidation state of +4.5 in a simple ionic picture. Our investigation of this $5 d$-based system is motivated by the fact that the fractional valence coupled with a geometrically frustrated lattice might lead to a spin-liquid state or possibly a heavy fermion state as in the $3 d$-based $\mathrm{LiV}_{2} \mathrm{O}_{4} \cdot{ }^{11}$ This however did not turn out to be the case. Whereas we confirmed the onset of long-range order below $4 \mathrm{~K}$ in $\mathrm{Ba}_{3} \mathrm{YIr}_{2} \mathrm{O}_{9}$ (in agreement with Ref. 10), we succeeded in suppressing the magnetic order with the application of pressure. In fact, when $\mathrm{Ba}_{3} \mathrm{YIr}_{2} \mathrm{O}_{9}$ was subjected to a pressure of $8 \mathrm{GPa}$ at $1273 \mathrm{~K}$, it transformed to a cubic double perovskite structure as evidenced from $\mathrm{x}$-ray diffraction under ambient conditions. Though the high-pressure (HP) synthesized sample remains insulating (based on our resistivity measurements), it has a metal-like linear heat capacity coefficient $\gamma=10 \mathrm{~mJ} / \mathrm{mol}-\mathrm{Ir} \mathrm{K}^{2}$. Further, the ${ }^{89} \mathrm{Y}$ nuclear magnetic resonance (NMR) shift is found to be independent of temperature $(T)$ below $120 \mathrm{~K}$ and the ${ }^{89} \mathrm{Y}$ NMR spin-lattice relaxation rate crosses over from $T$-independent behavior at high temperature to a linear $T$ dependence below about $45 \mathrm{~K}$. These results point to the existence of low-energy excitations at low temperatures. In the absence of metallic behavior in the resistivity and the presence of local moments, the low- $T$ data suggest the formation of an exotic ground state for the HP phase, possibly a gapless, quantum spin-liquid (QSL) state. The strong spin-orbit coupling (SOC) route as opposed to the geometrical frustration route has been suggested as a means of enhancing quantum fluctuations and the possible formation of a QSL in, say, double perovskites. ${ }^{12}$ The formation of a spin-orbital liquid has been suggested earlier in the Fe-based cubic spinel $\mathrm{FeSc}_{2} \mathrm{~S}_{4}$ where the magnetic atoms reside on the unfrustrated "A" sublattice. ${ }^{13-15}$

We have prepared a polycrystalline sample of $\mathrm{Ba}_{3} \mathrm{YIr}_{2} \mathrm{O}_{9}$ by the solid-state reaction method as detailed in Ref. 10 . Further, the ambient pressure (AP) sample was treated under $8 \mathrm{GPa}$ pressure at $1273 \mathrm{~K}$ for $30 \mathrm{~min}$ to get the HP sample. Due to the small size of the HP cell, only about $150 \mathrm{mg}$ of sample was obtained. Powder x-ray diffraction (XRD) measurements were performed at room temperature with $\mathrm{Cu}$ $K_{\alpha}$ radiation $(\lambda=1.54182 \AA)$ in a PANalytical X'Pert PRO diffractometer. Magnetization measurements were carried out in the temperature range 2-400 $\mathrm{K}$ and field range $0-7 \mathrm{~T}$ using a Quantum Design SQUID VSM. Heat capacity measurements were performed using the heat capacity attachment of a Quantum Design Physical Properties Measurement System 
(PPMS) in the temperature range 1.8-300 $\mathrm{K}$ and field range 0-9 T. By breaking a piece of hard pellet for both the AP and HP phase, we were able to find a few single crystal-like pieces with approximate size $600 \mu \mathrm{m} \times 150 \mu \mathrm{m} \times 100 \mu \mathrm{m}$. These pieces (later mentioned as single crystals) were harder than the rest of the pellet and looked shiny under the microscope. We have performed resistivity measurements on these single crystals using a home-built setup. For ${ }^{89} \mathrm{Y}$ NMR measurements, a Tecmag pulse spectrometer and a fixed magnetic field 93.954 kOe obtained inside a room-temperature-bore Varian superconducting magnet were used. Variable temperature was obtained with the help of an Oxford cryostat and accessories. The ${ }^{89} \mathrm{Y}$ nucleus has spin $I=1 / 2$ (100\% natural abundance) and gyromagnetic ratio $\gamma / 2 \pi=2.08583 \mathrm{MHz} / \mathrm{T}$.

Our XRD analysis confirmed that the AP sample was formed in a hexagonal structure $\left(\mathrm{P}_{3} / \mathrm{mmc}\right)$ as reported earlier ${ }^{10}$ but we found that after high-pressure treatment the structure changed to a cubic double perovskite $\left(\mathrm{Ba}_{2} \mathrm{MM}^{\prime} \mathrm{O}_{6}\right)$ phase with space group Fm-3m. It is relevant to mention that $\mathrm{Ba}_{3} \mathrm{NiSb}_{2} \mathrm{O}_{9}$ was also reported to transform from a hexagonal $\left(\mathrm{P}_{3} / \mathrm{mmc}\right)$ to cubic $(\mathrm{Fm}-3 \mathrm{~m})$ phase when treated under $9 \mathrm{GPa}$ pressure at $1273 \mathrm{~K}$ for $30 \mathrm{~min} .{ }^{16}$ In the XRD pattern (Fig. 1) of the HP phase, a few extra peaks (marked as *) are present which are identified as due to unreacted Ir and account for about $5 \%$ of the contribution. Also, all the major peaks have a shoulder on the right side as shown in the inset of Fig. 1. These shoulders possibly arise from a double perovskite phase with a slightly different cell parameter (this could happen due to a miscibility gap in the phase diagram). Accordingly, a two-phase Rietveld refinement (Fig. 1) performed with our XRD data yields lattice parameter $a=8.3248 \AA$ for the primary phase and $a^{\prime}=8.202 \AA$ for the secondary phase (about $10 \%$ content). A similar double perovskite $\mathrm{Ba}_{2} \mathrm{YIrO}_{6}$ (space group Fm-3m) has lattice parameter $a=8.35032 \AA .{ }^{17}$ Atomic cell parameters for the primary phase resulting from our refinement are listed in Table I. Attempts to explain

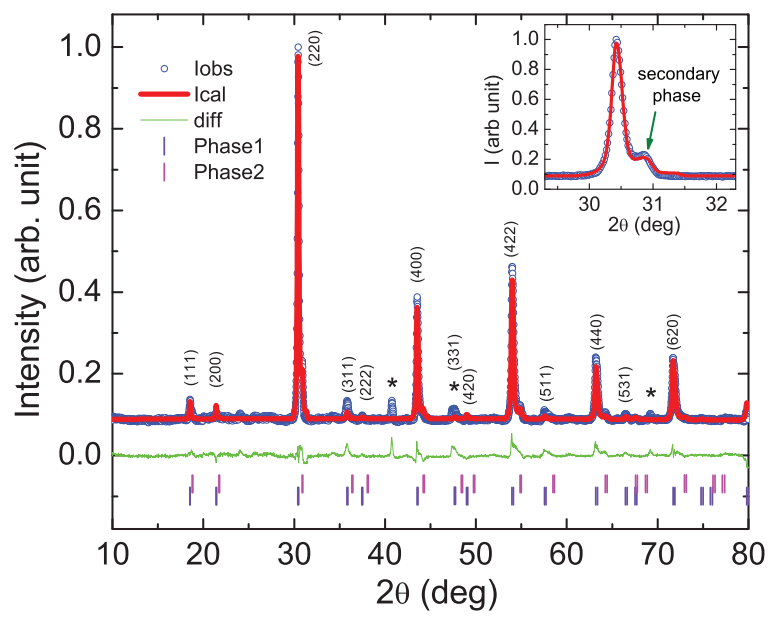

FIG. 1. (Color online) The x-ray diffraction pattern of the HP phase of $\mathrm{Ba}_{3} \mathrm{YIr}_{2} \mathrm{O}_{9}$ sample is shown along with a two-phase refinement with space group Fm-3m. The (hkl) positions are also indicated. A 5\% impurity phase of unreacted Ir is found. The peaks corresponding to Ir are marked with (*). Inset: The main peak of the primary phase is shown.
TABLE I. Atomic parameters obtained from refinement of x-ray powder diffraction pattern at room temperature for the primary phase of the HP sample.

\begin{tabular}{lccccc}
\hline \hline Atoms & Site & $X$ & $Y$ & $Z$ & Occupancy \\
\hline $\mathrm{Ba}(1)$ & $8 \mathrm{c}$ & 0.25 & 0.25 & 0.25 & 1 \\
$\mathrm{Y}(1)$ & $4 \mathrm{a}$ & 0 & 0 & 0 & 0.67 \\
$\mathrm{Ir}(1)$ & $4 \mathrm{a}$ & 0 & 0 & 0 & 0.33 \\
$\mathrm{Ir}(2)$ & $4 \mathrm{~b}$ & 0.5 & 0.5 & 0.5 & 1 \\
$\mathrm{O}(1)$ & $24 \mathrm{e}$ & $0.2615(4)$ & 0 & 0 & 1 \\
\hline \hline
\end{tabular}

the XRD data using a cubic perovskite structure [chemical formula $\left.\mathrm{Ba}\left(\mathrm{Y}_{1 / 3} \mathrm{Ir}_{2 / 3}\right) \mathrm{O}_{3}\right]$ with approximately half the lattice parameter of the double perovskite [chemical formula $\left.\mathrm{Ba}_{2} \operatorname{Ir}\left(\mathrm{Y}_{2 / 3} \mathrm{Ir}_{1 / 3}\right) \mathrm{O}_{6}\right]$ did not lead to a good refinement. On the other hand, we find that an ordered arrangement of $\mathrm{IrO}_{6}$ and $\mathrm{Y}_{2 / 3} \operatorname{Ir}_{1 / 3} \mathrm{O}_{6}$ octahedra form the cubic double perovskite $\mathrm{Ba}_{2} \operatorname{Ir}\left(\mathrm{Y}_{2 / 3} \operatorname{Ir}_{1 / 3}\right) \mathrm{O}_{6}$ structure of the HP phase (shown in Fig. 2). We note that every $\mathrm{Y}$ will have $6 \mathrm{Ir}$ as its nearest neighbors while as next-nearest neighbors it will have $33 \% \mathrm{Ir}$ and $67 \% \mathrm{Y}$.

Figure 3 provides an overview of our data pertaining to some basic measurements on $\mathrm{HP}$ and AP phases of $\mathrm{Ba}_{3} \mathrm{YIr}_{2} \mathrm{O}_{9}$. Resistivity data [shown in Fig. 3(a)] on single crystals show insulating behavior for both AP and HP samples. The AP sample shows an activated behavior with a gap of about $0.3 \mathrm{eV}$. For the HP sample, as well, the resistivity increases with decreasing temperature though with a weaker rise. In the heat capacity, on the other hand, a clear anomaly at about $4 \mathrm{~K}$ is seen for the AP sample [Fig. 3(b)], which suggests long-range order. The anomaly goes away in the HP sample indicating that the ordering has been suppressed by the application of pressure. It is further interesting to note that for the HP sample below about $10 \mathrm{~K}$, the heat capacity $C_{P}(T)$ is metal-like with a linear $C_{P} / T$ vs $T^{2}$ (with slope $\beta=0.44 \mathrm{~mJ} / \mathrm{mol}-\mathrm{Ir} \mathrm{K}^{4}$ ) and a nonzero $y$-axis intercept giving a Sommerfeld coefficient $\gamma=10 \mathrm{~mJ} / \mathrm{mole}-\mathrm{Ir}$ $\mathrm{K}^{2}$. The inset of Fig. 3(c) shows the magnetic susceptibility

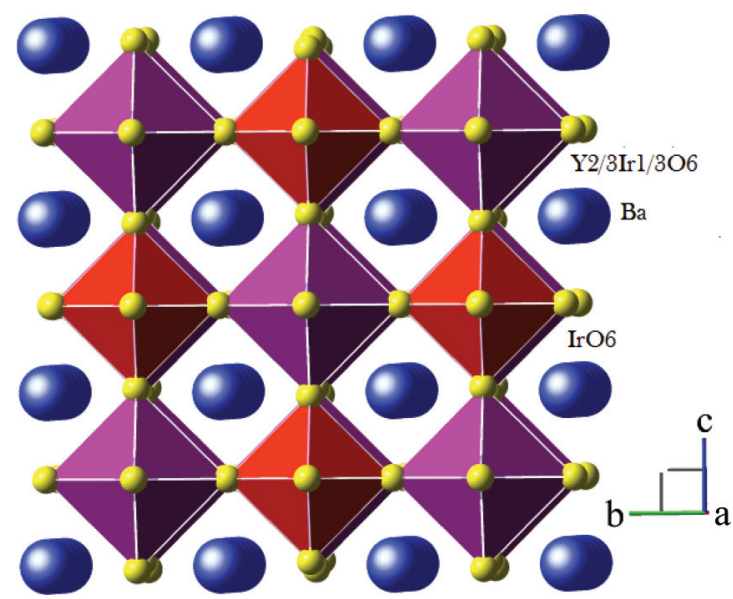

FIG. 2. (Color online) The crystal structure of the high-pressure synthesized cubic phase is shown. The blue and yellow atoms represent $\mathrm{Ba}$ and $\mathrm{O}$, respectively. The red octahedra are $\mathrm{IrO}_{6}$ octahedra while the violet octahedra contain Ir or $\mathrm{Y}$ at their centers. 

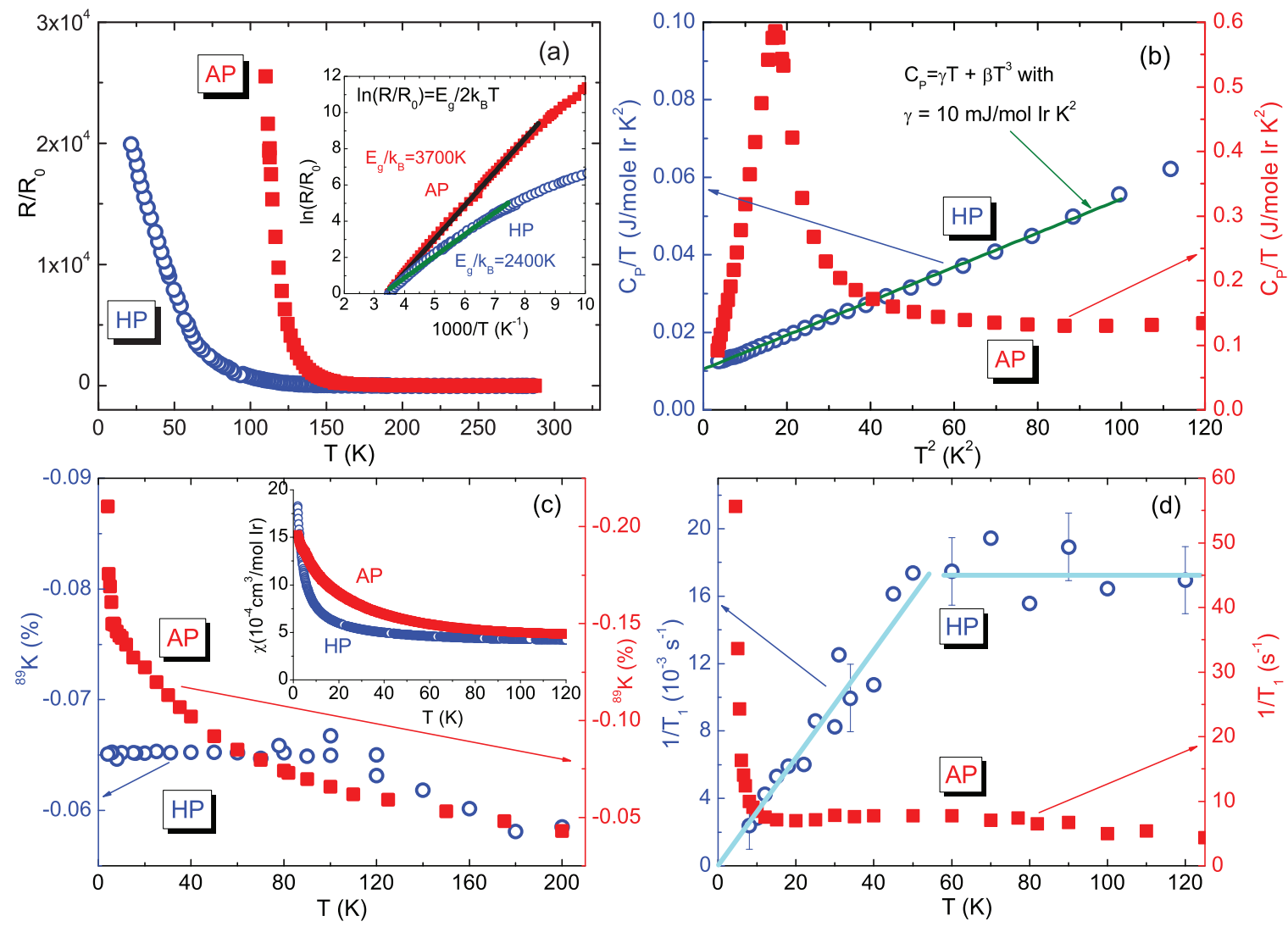

FIG. 3. (Color online) The results of different measurements on the HP sample (blue open circles) are shown in comparison with its AP counterpart (red solid squares). In case of (b), (c), and (d), AP sample corresponds to the right axis and HP sample to the left axis while for (a) both correspond to the left axis. (a) The normalized resistivity is shown as a function of temperature. Inset: $\ln \left(\mathrm{R} / \mathrm{R}_{0}\right)$ is plotted with $1000 / T$. The solid lines are fit with the formula mentioned in the figure. (b) The $C_{P} / T$ is shown as a function of $T^{2}$. The green solid line is a fit of the HP data with the formula stated in the figure. (c) Temperature variation of ${ }^{89} \mathrm{Y}$ NMR shift for the HP and AP samples are shown. Their bulk susceptibilities are shown in the inset. (d) The ${ }^{89} \mathrm{Y}$ spin-lattice relaxation rate $\left(1 / T_{1}\right)$ is shown as a function of temperature. The light blue solid lines are guides to eye.

$\chi(T)$ of the samples in a field of $5 \mathrm{kOe}$. For the AP sample, magnetic susceptibility shows a kink at $T \sim 4 \mathrm{~K}$ indicative of magnetic ordering as reported earlier. ${ }^{10}$ Susceptibility data of the AP sample could be fitted to the Curie law (with $\theta \sim 0$ ) in the temperature range $30-300 \mathrm{~K}$, which yields a $T$-independent susceptibility $\chi_{0}=3.8 \times 10^{-4} \mathrm{~cm}^{3} / \mathrm{mol} \mathrm{Ir}$ and a Curie constant $C=0.0125 \mathrm{~cm}^{3} \mathrm{~K} / \mathrm{mol}$ Ir. The small Curie term (nearly thirty times smaller than that for $S=1 / 2$ ) is not unusual in iridates. In fact many other iridates have been reported with small Curie terms. ${ }^{8,18,19}$ Below about $30 \mathrm{~K}$, the susceptibility deviates from Curie behavior presumably due to the building up of magnetic correlations. Consistent with the susceptibility behavior in the AP sample, the ${ }^{89} \mathrm{Y}$ NMR shift $(K)$ increases with a decrease in temperature [Fig. 3(c)]. One should note that whereas in the AP phase the $\theta_{\mathrm{CW}}$ is nearly zero from $\chi(T)$ data, a CW fit of $K(T)$ gives $\theta_{\mathrm{CW}} \approx-30 \mathrm{~K}^{20}$ For the HP sample, on the other hand, any susceptibility anomaly indicative of a magnetic ordering was not seen down to $2 \mathrm{~K}$ nor was there any ZFC-FC splitting observed. The $\chi(T)$ for the HP sample could be fitted to the CW law in almost the whole temperature range resulting in the parameters $\chi_{0}=3.9 \times 10^{-4} \mathrm{~cm}^{3} / \mathrm{mol} \mathrm{Ir}, C=0.0045 \mathrm{~cm}^{3} \mathrm{~K} / \mathrm{mol} \mathrm{Ir}$ and $\theta=-1.6 \mathrm{~K}$. For the HP sample, the $C$ value is smaller by a further factor of 3 compared to its AP counterpart while the $\chi_{0}$ is nearly unchanged. We have measured ${ }^{89} \mathrm{Y}$ NMR spectra for the HP sample at different temperatures as shown in Fig. 4. The spectra for the HP sample is very narrow $(\sim 2 \mathrm{kHz})$ and becomes broader with decreasing temperature. The full width at half maxima (FWHM) for the HP sample is shown as a function of temperature in the inset of Fig. 2 and compared with the FWHM for the AP sample. The temperature variation of FWHM for the HP sample is much weaker in comparison to the AP sample. However the increase in FWHM at low temperature probably suggests presence of small local moments in the HP sample. As shown in Fig. 3(c), the ${ }^{89}$ Y NMR shift (obtained from the peak position of individual spectra) for the HP sample increases with a decrease in temperature and becomes temperature independent below about $100 \mathrm{~K}$. The magnitude of the change in $K$ with temperature is five times smaller than for the AP sample. These points indicate that the magnetic moments in the HP sample are very weak. Once again, the small $\theta_{\mathrm{CW}}$ from $\chi(T)$ analysis need not suggest weak correlations but might simply suggest that the intrinsic moments are very small and that the bulk susceptibility data are dominated by extrinsic effects. The saturation of $K$ below $100 \mathrm{~K}$ suggests a quenching of the local moments and the small low- $T$ Curie term seen in the bulk susceptibility is likely extrinsic. 


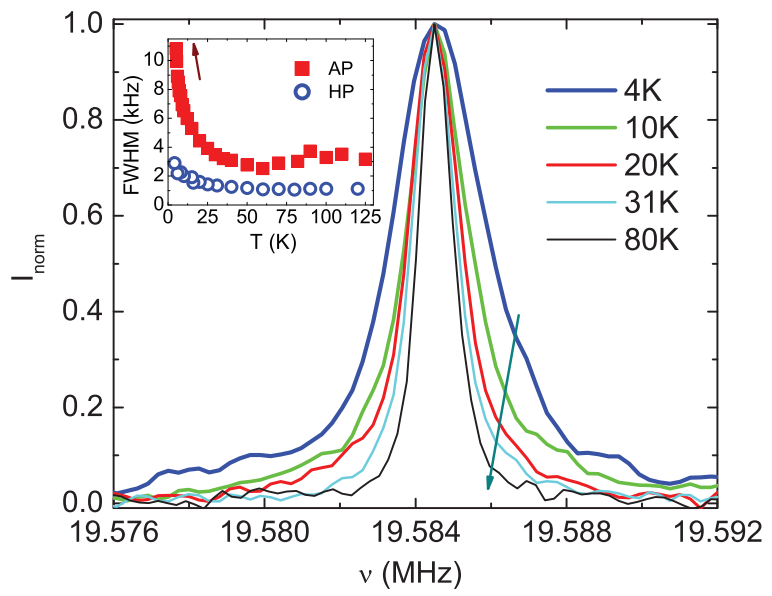

FIG. 4. (Color online) ${ }^{89} \mathrm{Y}$ NMR spectra at different temperatures for the HP sample are shown. The arrow indicates measurement temperature in increasing order. Inset: FWHM of the AP sample as well as the HP sample as functions of temperature. The arrow indicates that FWHM for the AP sample goes much higher at low temperature $(40 \mathrm{kHz}$ at $4 \mathrm{~K})$.

We next probe the low-energy excitations of both the AP and the HP sample using ${ }^{89}$ Y NMR spin-lattice relaxation rate measurements. We have measured the spin-lattice relaxation rate $\left(1 / T_{1}\right)$ using a standard saturation recovery method following a $\pi / 2-t-(\pi / 2-\pi)$ pulse sequence in the temperature range 4-300 K for the AP sample and 8-120 K for the HP sample. For the AP sample, nuclear magnetization recoveries are single exponential as expected for an $I=1 / 2$ nucleus. $^{20}$ The relaxation time $\left(T_{1}\right)$ for the AP sample varies from about $10 \mathrm{~ms}$ to about $400 \mathrm{~ms}$ as a function of temperature. For the HP sample, the nuclear magnetization recovery after a saturating pulse has an initial short component followed by a longer component. A few representative recovery data are shown in the Fig. 5. We have fitted them with the formula

$$
1-m(t) / m_{0}=A\left[B \exp \left(-t / T_{L}\right)+(1-B) \exp \left(-t / T_{S}\right)\right],
$$

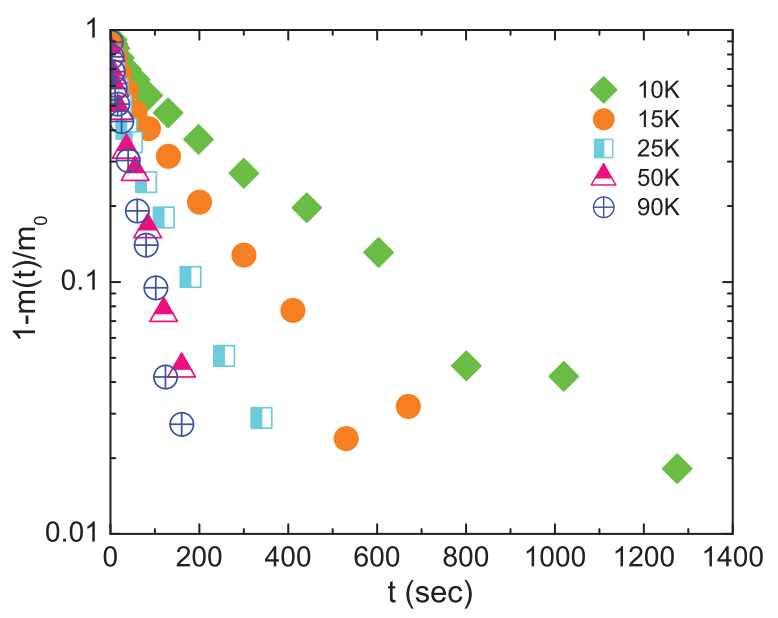

FIG. 5. (Color online) Shown here are some representative data for the HP sample for the recovery of the ${ }^{89} \mathrm{Y}$ longitudinal nuclear magnetization after a saturating pulse. where $T_{L}$ and $T_{S}$ are the longer and shorter components of $T_{1}$, respectively, and $B$ is $\sim 0.7$. The longer component $T_{L}$ varies from about $40 \mathrm{~s}$ at $100 \mathrm{~K}$ to about $400 \mathrm{~s}$ at $8 \mathrm{~K}$. Below $8 \mathrm{~K}, T_{L}$ becomes even longer though we did not take detailed data. The major differences of $T_{1}$ between the AP and HP sample are, (i) $T_{1}$ is almost three orders of magnitude higher for the HP sample compared to the AP sample and (ii) relaxation rate $\left(1 / T_{1}\right)$ increases with decreasing temperature for the AP sample but decreases with decreasing temperature $(T<45 \mathrm{~K})$ for the HP sample. A clear signature of ordering is seen in the divergence of the ${ }^{89} \mathrm{Y}$ NMR $1 / T_{1}$ for the AP sample [Fig. 3(d)]. In contrast, for the HP sample, $1 / T_{1}$ is $T$ independent at high $T$ as might be expected in a paramagnetic insulator but crosses over to a linear variation below about $45 \mathrm{~K}$ [the rate corresponding to the longer $T_{1}$ component $\left(1 / T_{L}\right)$ is plotted here].

To summarize, whereas the AP sample shows unambiguous evidence of being a magnetic insulator, the data on the HP sample gives apparently conflicting signals. Whereas the low- $T$ NMR shift (and hence the intrinsic susceptibility), heat capacity, and $1 / T_{1}$ are all metal-like, the resistivity (measured on a single crystal) is insulating. We suggest that these results imply the exotic possibility of the absence of charge excitations while spin excitations are present. The linear contribution to the low- $T$ heat capacity as also to the ${ }^{89} \mathrm{Y} 1 / T_{1}$ then come from excitations from a spinon Fermi surface as might happen in a gapless QSL. In this context it is relevant to point out NMR results on other spin liquid systems. For the $5 d$-based spin liquid system $\mathrm{Na}_{4} \mathrm{Ir}_{3} \mathrm{O}_{8},{ }^{23} \mathrm{Na} \mathrm{NMR}$ by Takagi et $a .^{21}$ finds a negligible temperature dependence of shift below about $100 \mathrm{~K}$ reflecting the independence from temperature of the intrinsic susceptibility. In this case the ${ }^{23} \mathrm{Na}$ $1 / T_{1}$ was constant above $200 \mathrm{~K}$ below which it followed a power law with power 1 in the range $2-10 \mathrm{~K}$ and with power 2.5 in the temperature range $100-200 \mathrm{~K}$. They attributed this low-temperature linear behavior to low-lying spin excitations. Itou et al $^{22}$ also found almost no temperature dependence of shift in their ${ }^{13} \mathrm{C}$ NMR data down to $18.7 \mathrm{mK}$ on the organic system $\mathrm{EtMe}_{3} \mathrm{Sb}\left[\mathrm{Pd}(\mathrm{dmit})_{2}\right]_{2}$ with a spin-liquid ground state while $1 / T_{1}$ was found to be constant in some range above $1 \mathrm{~K}$ and varied as $T^{2}$ below $1 \mathrm{~K}$. In the organic spin liquid system $\kappa$-(BEDT-TTF $)_{2} \mathrm{Cu}_{2}(\mathrm{CN})_{3}$, Shimizu et al. ${ }^{23}$ found no change in ${ }^{1} \mathrm{H}$ NMR spectral position from $36.1 \mathrm{~K}$ down to $32 \mathrm{mK}$ while $1 / T_{1}$ followed a power law at low temperature, from which the authors concluded that the ground state has gapless low-lying spin excitations. Our NMR data are qualitatively similar to the cases listed above, providing further justification for the spin liquid state at low $T$. It is worth mentioning that another B-site ordered double perovskite $\mathrm{Ba}_{2} \mathrm{YMoO}_{6}$ was reported to have an exotic valence bond glass state ${ }^{24}$ or a collective (gapped) spin-singlet state ${ }^{25}$ whereas Ref. 12 suggested a quadrupolar ordered state.

In conclusion, we have presented evidence based on bulk probes (resistivity, susceptibility, heat capacity) and local probes $\left({ }^{89} \mathrm{Y}\right.$ NMR shift and $\left.1 / T_{1}\right)$ for a possible gapless QSL state in the HP (cubic double perovskite) phase of $\mathrm{Ba}_{3} \mathrm{YIr}_{2} \mathrm{O}_{9}$. The occurrence of a spin-liquid state even for a geometrically nonfrustrated lattice suggests the presence of anisotropic exchange interactions strongly tied to the large spin-orbit coupling in this $5 d$-based system. The AP phase on 
the other hand was found to order magnetically around $4 \mathrm{~K}$. A strong SOC likely plays a crucial role in the insulating behavior of both AP and HP samples.

We thank the Department of Science and Technology, Government of India, for financial support. This work was partially supported by Swiss National Science Foundation and the Indo Swiss Joint Research Programme. F.C.C. acknowledges the support from National Science Council of Taiwan. We thank B. H. Chen for his help in preparation of the HP phase sample. We also thank S. K. Panda, I. Dasgupta, Kedar Damle, and P. P. Singh for useful discussions.
*Present address: Leibniz-Institute for Solid State and Materials Research, IFW Dresden, 01171 Dresden, Germany.

†mahajan@phy.iitb.ac.in

${ }^{1}$ J. G. Bednorz and K. A. Mueller, Z. Phys. B 64, 189 (1986).

${ }^{2}$ Y. Kamihara, T. Watanabe, M. Hirano, and H. Hosono, J. Am. Chem. Soc. 130, 3296 (2008).

${ }^{3}$ Y. Tokura, Colossal Magnetoresistive Oxides (Gordon \& Breach Science, New York, 2000).

${ }^{4}$ Y. Okamoto, M. Nohara, H. Aruga-Katori, and H. Takagi, Phys. Rev. Lett. 99, 137207 (2007).

${ }^{5}$ B. J. Kim, H. Jin, S. J. Moon, J.-Y. Kim, B.-G. Park, C. S. Leem, J. Yu, T. W. Noh, C. Kim, S.-J. Oh, J.-H. Park, V. Durairaj, G. Cao, and E. Rotenberg, Phys. Rev. Lett. 101, 076402 (2008).

${ }^{6}$ B. J. Kim, H. Ohsumi, T. Komesu, S. Sakai, T. Morita, H. Takagi, and T. Arima, Science 323, 1329 (2009).

${ }^{7}$ Y. Singh and P. Gegenwart, Phys. Rev. B 82, 064412 (2010).

${ }^{8}$ T. Dey, A. V. Mahajan, P. Khuntia, M. Baenitz, B. Koteswararao, and F. C. Chou, Phys. Rev. B 86, 140405(R) (2012).

${ }^{9}$ M. Becker, M. Hermanns, B. Bauer, M. Garst, and S. Trebst (unpublished).

${ }^{10}$ Y. Doi and Y. Hinatsu, J. Phys.: Condens. Matter 16, 2849 (2004).

${ }^{11}$ S. Kondo, D. C. Johnston, C. A. Swenson, F. Borsa, A. V. Mahajan, L. L. Miller, T. Gu, A. I. Goldman, M. B. Maple, D. A. Gajewski, E. J. Freeman, N. R. Dilley, R. P. Dickey, J. Merrin, K. Kojima, G. M. Luke, Y. J. Uemura, O. Chmaissem, and J. D. Jorgensen, Phys. Rev. Lett. 78, 3729 (1997).

${ }^{12}$ G. Chen, R. Pereira, and L. Balents, Phys. Rev. B 82, 174440 (2010).
${ }^{13}$ N. Buettgen, J. Hemberger, V. Fritsch, A. Krimmel, M. Muecksch, H.-A. K. von Nidda, P. Lunkenheimer, R. Fichtl, V. Tsurkan, and A. Loidl, New J. Phys. 6, 191 (2004).

${ }^{14}$ V. Fritsch, J. Hemberger, N. Buettgen, E.-W. Scheidt, H.-A. Krug von Nidda, A. Loidl, and V. Tsurkan, Phys. Rev. Lett. 92, 116401 (2004).

${ }^{15}$ A. Krimmel, M. Muecksch, V. Tsurkan, M. M. Koza, H. Mutka, and A. Loidl, Phys. Rev. Lett. 94, 237402 (2005).

${ }^{16}$ J. G. Cheng, G. Li, L. Balicas, J. S. Zhou, J. B. Goodenough, Cenke Xu, and H. D. Zhou, Phys. Rev. Lett. 107, 197204 (2011).

${ }^{17}$ W. T. Fu and D. J. W. IJdo, J. Alloy Comp. 394, L5 (2005).

${ }^{18}$ G. Cao, J. Bolivar, S. McCall, J. E. Crow, and R. P. Guertin, Phys. Rev. B 57, R11039 (1998).

${ }^{19}$ P. J. Baker, J. S. Möller, F. L. Pratt, W. Hayes, S. J. Blundell, T. Lancaster, T. F. Qi, and G. Cao, Phys. Rev. B 87, 180409(R) (2013).

${ }^{20}$ T. Dey, R. Kumar, A. V. Mahajan, S. D. Kaushik, and V. Siruguri (unpublished).

${ }^{21}$ See http://online.itp.ucsb.edu/online/motterials07/takagi

${ }^{22}$ T. Itou, K. Yamashita, M. Nishiyama, A. Oyamada, S. Maegawa, K. Kubo, and R. Kato, Phys. Rev. B 84, 094405 (2011).

${ }^{23}$ Y. Shimizu, K. Miyagawa, K. Kanoda, M. Maesato, and G. Saito, Phys. Rev. Lett. 91, 107001 (2003).

${ }^{24}$ M. A. de Vries, A. C. Mclaughlin, and J.-W. G. Bos, Phys. Rev. Lett. 104, 177202 (2010).

${ }^{25}$ T. Aharen, J. E. Greedan, C. A. Bridges, A. A. Aczel, J. Rodriguez, G. MacDougall, G. M. Luke, T. Imai, V. K. Michaelis, S. Kroeker, H. Zhou, C. R. Wiebe, and L. M. D. Cranswick, Phys. Rev. B 81, 224409 (2010). 